TAIWANESE JOURNAL OF MATHEMATICS

Vol. 19, No. 1, pp. 31-49, February 2015

DOI: $10.11650 /$ tjm.19.2015.4323

This paper is available online at http://journal.taiwanmathsoc.org.tw

\title{
PROPERTIES OF DUAL TOEPLITZ OPERATORS WITH APPLICATIONS TO HAPLITZ PRODUCTS ON THE HARDY SPACE OF THE POLYDISK
}

\author{
Lakhdar Benaissa* and Hocine Guediri
}

\begin{abstract}
In this paper, we introduce dual Toeplitz operators on the orthogonal complement of the Hardy space of the polydisk and establish their main algebraic properties using an auxiliary transformation of operators. As a byproduct, we exploit this mysterious transformation in the investigation of boundedness and compactness of Hankel products and mixed Toeplitz-Hankel products on the Hardy space of the polydisk.
\end{abstract}

\section{INTRODUCTION}

Dual Toeplitz operators on the orthogonal complements of various Hilbert spaces of analytic functions become nowadays among the concrete classes of operators that attract attention of operator theorists. Algebraic and spectral properties of these operators in different contexts have been the subject of extensive studies in the last decade. For a detailed account on this topic we refer to $[4,7,9,14,17]$ and the references therein.

The purpose of this paper is two fold. First, to outline some basic algebraic properties of dual Toeplitz operators in the setting of the Hardy space of the polydisk. In particular, in Section 3, we characterize commuting dual Toeplitz operators as well as normal ones. The commutativity task in related contexts has been considered in $[2,3,6,10,12,14,16,17]$.

Furthermore, in Section 4, we investigate products of dual Toeplitz operators. More precisely, we establish Brown-Halmos type theorems and exploit them to characterize the zero divisors among dual Toeplitz operators as well as symbols giving rise to isometric, idempotent and unitary dual Toeplitz operators. These facts in related settings can be found in $[1,3,6,10,16,17]$; and our approach is similar to that used in [14].

Received January 15, 2014, accepted April 18, 2014.

Communicated by Yong-Sheng Han.

2010 Mathematics Subject Classification: 47B35.

Key words and phrases: Dual Toeplitz operator, Hardy space of the polydisk, Commuting, Brown-Halmos, Hankel products, Mixed Toeplitz-Hankel products.

*Corresponding author. 
All the above results hinge on a crucial transformation of operators (which goes back to Stroethoff \& Zheng [17]), namely the operator $\mathcal{S}_{w}$ constructed in Section 2 (see formula (2.11)), which proves very suitable for such purposes. In the setting of the Bergman space of the polydisk, an analog operator is already present in force in the work of Y.F. Lu and S.X. Shang [14]. This transformation reveals an interesting characterization of dual Toeplitz operators that is closely related to the intertwining relations of such operators in one dimension, see [3, 7, 10]. For a brief history of this powerful transformation we refer to [7].

Second, to study Hankel products and mixed Toeplitz-Hankel products on the Hardy space of the polydisk. In particular, in Section 5, we make use of this pioneering operator $\mathcal{S}_{w}$ in order to establish necessary conditions for boundedness and compactness of these products. Products of merely Toeplitz operators in the present setting have been considered by Ding in [5]; for the same problem in related contexts we refer to $[8,11,13]$ and the references therein.

First, let us start with setting up the framework of our study as well as the construction and the main properties of the indispensable operator $\mathcal{S}_{w}$.

\section{Preliminaries}

Let $\mathbb{D}$ be the unit disk in the complex plane $\mathbb{C}$, and let $\mathbb{T}=\partial \mathbb{D}$ be its boundary (the unit circle). For $n \geq 1$, the polydisk $\mathbb{D}^{n}$ and its distinguished (or shilov) boundary $\mathbb{T}^{n}$, (the $n$-torus), are respectively the cartesian products of $n$ copies of $\mathbb{D}$ and $\mathbb{T}$; they are defined respectively by

$$
\begin{aligned}
& \mathbb{D}^{n}:=\left\{z=\left(z_{1}, z_{2}, \ldots, z_{n}\right) \in \mathbb{C}^{n},\left|z_{j}\right|<1, j=1, \ldots, n\right\}, \\
& \mathbb{T}^{n}:=\left\{\zeta=\left(\zeta_{1}, \zeta_{2}, \ldots, \zeta_{n}\right) \in \mathbb{C}^{n},\left|\zeta_{j}\right|=1, j=1, \ldots, n\right\} .
\end{aligned}
$$

Let $d \sigma(\zeta)$ be the normalized Haar measure on $\mathbb{T}^{n}$; it is obtained as the product of the normalized Lebesgue measure on $\mathbb{T}$, i.e. $d \sigma(\zeta)=\frac{d \theta_{1} d \theta_{2} \ldots d \theta_{n}}{(2 \pi)^{n}}$ where $\zeta_{j}=e^{i \theta_{j}}, j=$ $1, \ldots, n$. Thus, the Lebesgue space $L^{1}\left(\mathbb{T}^{n}, d \sigma\right)$ is defined in the customary way, and we have

$$
\int_{\mathbb{T}^{n}} f(\zeta) d \sigma(\zeta)=\frac{1}{(2 \pi)^{n}} \int_{0}^{2 \pi} \ldots \int_{0}^{2 \pi} f\left(e^{i \theta_{1}} e^{i \theta_{2}}, \ldots, e^{i \theta_{n}}\right) d \theta_{1} d \theta_{2} \ldots d \theta_{n} .
$$

The Hardy space of the polydisk $\mathcal{H}^{2}\left(\mathbb{D}^{n}\right)$ is defined to be the set of all holomorphic functions $f: \mathbb{D}^{n} \rightarrow \mathbb{C}$ satisfying:

$$
\|f\|_{2}:=\left(\sup _{0<r<1} \int_{\mathbb{T}^{n}}|f(r \zeta)|^{2} d \sigma(\zeta)\right)^{\frac{1}{2}}<\infty .
$$

Recall that for every function $f \in \mathcal{H}^{2}\left(\mathbb{D}^{n}\right)$, the radial $\operatorname{limit}_{\lim } \operatorname{lit}_{r-} f(r \zeta)$ exists for almost every $\zeta \in \mathbb{T}^{n}$. Denoting this radial limit again by $f(\zeta)$, the Hardy space 
$\mathcal{H}^{2}\left(\mathbb{D}^{n}\right)$ can be regarded as a closed subspace of $L^{2}\left(\mathbb{T}^{n}, d \sigma\right)=L^{2}\left(\mathbb{T}^{n}\right)$. In fact if $\mathcal{H}^{2}\left(\mathbb{T}^{n}\right)$ denotes the closure of analytic polynomials in $L^{2}\left(\mathbb{T}^{n}\right)$, then every function in $\mathcal{H}^{2}\left(\mathbb{T}^{n}\right)$ can be identified with its holomorphic extension to $\mathbb{D}^{n}$ via the poisson extension; and hence we use the same notation for $f \in \mathcal{H}^{2}\left(\mathbb{T}^{n}\right)$ and its holomorphic extension $f \in \mathcal{H}^{2}\left(\mathbb{D}^{n}\right)$ to $\mathbb{D}^{n}$. For more details on the function theory in the polydisk we refer to Rudin's book [15]. Let us denote the orthogonal complement of the Hardy space $\mathcal{H}^{2}\left(\mathbb{T}^{n}\right)$ in $L^{2}\left(\mathbb{T}^{n}\right)$ by $\left(\mathcal{H}^{2}\left(\mathbb{T}^{n}\right)\right)^{\perp}$. It is well-known that

$$
\mathcal{H}^{2}\left(\mathbb{T}^{n}\right) \cong \mathcal{H}^{2}(\mathbb{T}) \otimes \mathcal{H}^{2}(\mathbb{T}) \otimes \ldots \otimes \mathcal{H}^{2}(\mathbb{T}),
$$

and that a similar factorization holds for $L^{2}\left(\mathbb{T}^{n}\right)$ as well. Thus clearly, for $n>1$, we would get $\left(\mathcal{H}^{2}\left(\mathbb{T}^{n}\right)\right)^{\perp} \supsetneqq \overline{\mathcal{H}_{0}^{2}\left(\mathbb{T}^{n}\right)}$, which motivates the study of dual Toeplitz operators on this space, (see Sec.1 of [7] for an explanation). The Hilbert space $\mathcal{H}^{2}\left(\mathbb{D}^{n}\right)$ is readily seen to be a functional Hilbert space with reproducing kernel given for $w=$ $\left(w_{1}, \ldots, w_{n}\right) \in \mathbb{D}^{n}$ by:

$$
K_{w}(z)=\prod_{j=1}^{n} \frac{1}{1-\bar{w}_{j} z_{j}}, \quad z=\left(z_{1}, \ldots, z_{n}\right) \in \mathbb{D}^{n} .
$$

Thus, the reproducing kernel of $\mathcal{H}^{2}\left(\mathbb{T}^{n}\right)$ reads as:

$$
K_{w}(\zeta)=\prod_{j=1}^{n} \frac{1}{1-\bar{w}_{j} \zeta_{j}}, \quad \zeta=\left(\zeta_{1}, \ldots, \zeta_{n}\right) \in \mathbb{T}^{n},
$$

while the normalized reproducing kernel is given by

$$
k_{w}(\zeta)=\prod_{j=1}^{n} \frac{\sqrt{1-\left|w_{j}\right|^{2}}}{1-\bar{w}_{j} \zeta_{j}}, \quad \zeta=\left(\zeta_{1}, \ldots, \zeta_{n}\right) \in \mathbb{T}^{n} .
$$

Let $\mathcal{P}$ denote the orthogonal projection from $L^{2}\left(\mathbb{T}^{n}\right)$ onto its closed subspace $\mathcal{H}^{2}\left(\mathbb{T}^{n}\right)$. For a symbol $\varphi \in L^{2}\left(\mathbb{T}^{n}\right)$, the Toeplitz operator on $\mathcal{H}^{2}\left(\mathbb{T}^{n}\right)$ is defined as follows:

$$
\begin{aligned}
T_{\varphi}: \mathcal{H}^{2}\left(\mathbb{T}^{n}\right) & \longrightarrow \mathcal{H}^{2}\left(\mathbb{T}^{n}\right) \\
f & \longmapsto T_{\varphi} f=\mathcal{P}(\varphi f) .
\end{aligned}
$$

Similarly, the "big" Hankel operator is defined by

$$
\begin{aligned}
H_{\varphi}: \mathcal{H}^{2}\left(\mathbb{T}^{n}\right) & \longrightarrow\left(\mathcal{H}^{2}\left(\mathbb{T}^{n}\right)\right)^{\perp} \\
f & \longmapsto H_{\varphi} f=\mathcal{Q}(\varphi f),
\end{aligned}
$$

where $\mathcal{Q}=I-\mathcal{P}$ is the orthogonal projection from $L^{2}\left(\mathbb{T}^{n}\right)$ onto $\left(\mathcal{H}^{2}\left(\mathbb{T}^{n}\right)\right)^{\perp}$.

A dual Toeplitz operator with symbol $\varphi \in L^{\infty}\left(\mathbb{T}^{n}\right)$ is defined to be a multiplication followed by a projection in the following way: 


$$
\begin{aligned}
S_{\varphi}:\left(\mathcal{H}^{2}\left(\mathbb{T}^{n}\right)\right)^{\perp} & \longrightarrow\left(\mathcal{H}^{2}\left(\mathbb{T}^{n}\right)\right)^{\perp} \\
f & \longmapsto S_{\varphi} f=\mathcal{Q}(\varphi f) .
\end{aligned}
$$

Since the projection $\mathcal{Q}$ has norm 1 , then $\left\|S_{\varphi}(f)\right\|_{2} \leq\|\varphi\|_{\infty}\|f\|_{2}, \forall f \in\left(\mathcal{H}^{2}\left(\mathbb{T}^{n}\right)\right)^{\perp}$.

Using the decomposition $L^{2}\left(\mathbb{T}^{n}\right)=\mathcal{H}^{2}\left(\mathbb{T}^{n}\right) \oplus\left(\mathcal{H}^{2}\left(\mathbb{T}^{n}\right)\right)^{\perp}$, for $f, g \in L^{\infty}\left(\mathbb{T}^{n}\right)$, the product equation $M_{f g}=M_{f} M_{g}$ can be written in matrix form as follows:

$$
\left(\begin{array}{cc}
T_{f g} & \mathcal{H}_{\bar{f} \bar{g}}^{*} \\
\mathcal{H}_{f g} & S_{f g}
\end{array}\right)=\left(\begin{array}{cc}
T_{f} & \mathcal{H}_{\bar{f}}^{*} \\
\mathcal{H}_{f} & S_{f}
\end{array}\right)\left(\begin{array}{cc}
T_{g} & \mathcal{H}_{\bar{g}}^{*} \\
\mathcal{H}_{g} & S_{g}
\end{array}\right),
$$

which yields the following algebraic equations:

$$
\begin{gathered}
T_{f g}=T_{f} T_{g}+H_{\bar{f}}^{*} H_{g}, \\
\mathcal{S}_{f g}=H_{f} H_{\bar{g}}^{*}+\mathcal{S}_{f} \mathcal{S}_{g}, \\
H_{f g}=H_{f} T_{g}+\mathcal{S}_{f} H_{g} .
\end{gathered}
$$

It follows that the commutator $\left[\mathcal{S}_{f}, \mathcal{S}_{g}\right]=\mathcal{S}_{f} \mathcal{S}_{g}-\mathcal{S}_{g} \mathcal{S}_{f}$ can be written as

$$
\left[\mathcal{S}_{f}, \mathcal{S}_{g}\right]=H_{g} H_{f}^{*}-H_{f} H_{\bar{g}}^{*}
$$

Since the "big" Hankel operator is trivial if the symbol is analytic, such identities reduce to:

Lemma 2.1. Let $f \in \mathcal{H}^{\infty}\left(\mathbb{T}^{n}\right)$ and $g \in L^{\infty}\left(\mathbb{T}^{n}\right)$, then we have

(i) $H_{g} T_{f}=\mathcal{S}_{f} H_{g}$.

(ii) $T_{\bar{f}} H_{g}^{*}=H_{g}^{*} \mathcal{S}_{\bar{f}}$.

(iii) $\mathcal{S}_{f g}=\mathcal{S}_{f} \mathcal{S}_{g}$.

(iv) $\mathcal{S}_{g \bar{f}}=\mathcal{S}_{g} \mathcal{S}_{\bar{f}}$.

For $\lambda \in \mathbb{D}$, let $\varphi_{\lambda}$ be the fractional linear transformation on $\mathbb{D}$ given by $\varphi_{\lambda}(u)=$ $\frac{\lambda-u}{1-\bar{\lambda} u}, u \in \mathbb{D}$. Each map $\varphi_{\lambda}$ is a disk automorphism and satisfies $\varphi_{\lambda}^{-1}=\varphi_{\lambda}$. For $\tau \in \mathbb{T}$, the mapping $\varphi_{\lambda}(\tau)=\frac{\lambda-\tau}{1-\bar{\lambda} \tau}$ remains well-defined on the circle $\mathbb{T}$, and moreover one has $\left|\varphi_{\lambda}(\tau)\right|=1$. Thus, for $w=\left(w_{1}, \ldots, w_{n}\right) \in \mathbb{D}^{n}$, the mapping $\varphi_{w}(\zeta)=\left(\varphi_{w_{1}}\left(\zeta_{1}\right), \ldots, \varphi_{w_{n}}\left(\zeta_{n}\right)\right), \zeta=\left(\zeta_{1}, \ldots, \zeta_{n}\right) \in \mathbb{T}^{n}$ is still well-defined on the n-torus $\mathbb{T}^{n}$, and $\varphi_{w} \circ \varphi_{w}$ is the identity map; for more details we refer to [15].

For $f$ and $g$ in $L^{2}\left(\mathbb{T}^{n}\right)$, consider the rank one operator defined by $(f \otimes g) h=<$ $h, g>f, \forall f \in L^{2}\left(\mathbb{T}^{n}\right)$; and note that $\|f \otimes g\|=\|f\|\|g\|$. Moreover, the unitary operator $\mathbb{U}_{w}$ is defined on $L^{2}\left(\mathbb{T}^{n}\right)$ by

$$
\mathbb{U}_{w} f=\left(f \circ \varphi_{w}\right) k_{w} .
$$


Thus, for a Toeplitz operator on $\mathcal{H}^{2}\left(\mathbb{T}^{n}\right)$, we have

$$
\mathbb{U}_{w} T_{f} \mathbb{U}_{w}=T_{f \circ \varphi_{w}} .
$$

For a multi-index $\alpha=\left(\alpha_{1}, \ldots, \alpha_{n}\right) \in \mathbb{N}^{n}$, recall that

$$
|\alpha|=\alpha_{1}+\ldots+\alpha_{n} ; \alpha !=\alpha_{1} ! \ldots \alpha_{n} ! ; z^{\alpha}=z_{1}^{\alpha_{1}} \ldots z_{n}^{\alpha_{n}} .
$$

We know that

$$
\prod_{j=1}^{n}\left(1-z_{j} \bar{w}_{j}\right)^{m}=\sum_{j=1}^{n} \sum_{\alpha_{j}=0}^{m} C_{m, \alpha} z^{\alpha} \bar{w}^{\alpha},
$$

where $C_{m, \alpha}=(-1)^{|\alpha|}\left(\begin{array}{c}m \\ \alpha_{1}\end{array}\right) \ldots\left(\begin{array}{c}m \\ \alpha_{n}\end{array}\right), \quad\left(\begin{array}{c}m \\ \alpha_{j}\end{array}\right)=\frac{m !}{\alpha_{j} !\left(m-\alpha_{j}\right) !}$.

In particular, we obtain

$$
K_{w}^{-1}(z)=\prod_{j=1}^{n}\left(1-z_{j} \bar{w}_{j}\right)=\sum_{|\alpha|=0}^{n}(-1)^{|\alpha|} z^{\alpha} \bar{w}^{\alpha} .
$$

Finally, for operators $\mathbf{T}$ and $\mathbf{S}$, we can easily verify that:

$$
\mathbf{T}(f \otimes g) \mathbf{S}^{*}=\mathbf{T} f \otimes \mathbf{S} g .
$$

Gluing all this stuff together, we arrive at the following crucial representation, whose proof is similar to that of Lemma 1.2 in [14]:

Proposition 2.1. On the Hardy space of the polydisk $\mathcal{H}^{2}\left(\mathbb{T}^{n}\right)$, the following operator identity holds:

$$
k_{w} \otimes k_{w}=\sum_{|\alpha|=0}^{n}(-1)^{|\alpha|} T_{\varphi_{w}^{\alpha}} T_{\overline{\varphi_{w}}}^{\alpha}, \forall w \in \mathbb{D}^{n} .
$$

Proof. Let $f$ be in $\mathcal{H}^{\infty}\left(\mathbb{D}^{n}\right)$. The invariant mean value property implies that

$$
f(0)=\int_{\mathbb{D}^{n}} f(w) d A(w) .
$$

Inserting $K_{w}(z) K_{w}^{-1}(z)$ and observing that $f(0)=(1 \otimes 1) f$, we get

$$
(1 \otimes 1) f=\int_{\mathbb{D}^{n}} K_{w}^{-1}(z) K_{w}(z) f(w) d A(w) .
$$

Using (2.5), we obtain 
$(1 \otimes 1) f=\sum_{|\alpha|=0}^{n}(-1)^{|\alpha|} z^{\alpha} \int_{\mathbb{D}^{n}} \bar{w}^{\alpha} \overline{K_{z}(w)} f(w) d A(w)=\sum_{|\alpha|=0}^{n}(-1)^{|\alpha|} z^{\alpha}\left(T_{\bar{w}^{\alpha}} f\right)(z)$.

Thus, we arrive at the following operator identity in $\mathcal{H}^{\infty}\left(\mathbb{D}^{n}\right)$ :

$$
(1 \otimes 1)=\sum_{|\alpha|=0}^{n}(-1)^{|\alpha|} T_{z^{\alpha}} T_{\bar{z}^{\alpha}} .
$$

Making appeal to the unitary operator $\mathbb{U}_{w}$, we get

$$
\mathbb{U}_{w}(1 \otimes 1) \mathbb{U}_{w}=\sum_{|\alpha|=0}^{n}(-1)^{|\alpha|}\left(\mathbb{U}_{w} T_{z^{\alpha}} \mathbb{U}_{w}\right)\left(\mathbb{U}_{w} T_{\bar{z}^{\alpha}} \mathbb{U}_{w}\right) .
$$

By (2.3) and the fact that $\mathbb{U}_{w} 1=k_{w}$, we obtain

$$
\mathbb{U}_{w}(1 \otimes 1) \mathbb{U}_{w}=\left(\mathbb{U}_{w} 1\right) \otimes\left(\mathbb{U}_{w} 1\right)=k_{w} \otimes k_{w} .
$$

Now, (2.4), (2.9) and (2.10) yield the following operator identity on $\mathcal{H}^{2}\left(\mathbb{D}^{n}\right)$ :

$$
k_{w} \otimes k_{w}=\sum_{|\alpha|=0}^{n}(-1)^{|\alpha|} T_{\varphi_{w}^{\alpha}} T_{\overline{\varphi_{w}}}^{\alpha}, \forall w \in \mathbb{D}^{n},
$$

which is valid also on $\mathcal{H}^{2}\left(\mathbb{T}^{n}\right)$ as well.

The latter key assertion gives rise to the following primordial operator transformation: for a bounded linear operator $T$ on $\left(\mathcal{H}^{2}\left(\mathbb{T}^{n}\right)\right)^{\perp}$ and $w \in \mathbb{D}^{n}$, define the linear operator $\mathcal{S}_{w}(T)$ by

$$
\mathcal{S}_{w}(T)=\sum_{|\alpha|=0}^{n}(-1)^{|\alpha|} \mathcal{S}_{\varphi_{w}^{\alpha}} T \mathcal{S}_{\bar{\varphi}_{w}}^{\alpha} .
$$

For a brief history of such type of transformations, we refer to [7].

Remark 2.1. For $n=1$ we recover the operator identity obtained in [10].

The operator $\mathcal{S}_{w}$ provides a nice characterization to our dual Toeplitz operators:

Proposition 2.2. If $\mathcal{S}_{f}$ is a dual Toeplitz operator on $\left(\mathcal{H}^{2}\left(\mathbb{T}^{n}\right)\right)^{\perp}$, then

$$
\mathcal{S}_{w}\left(\mathcal{S}_{f}\right)=0, \text { for all } w \in \mathbb{D}^{n} .
$$


Proof. $\quad$ Let $w \in \mathbb{D}^{n}$ and consider a dual Toeplitz operator $\mathcal{S}_{f}$ on $\left(\mathcal{H}^{2}\left(\mathbb{T}^{n}\right)\right)^{\perp}$, with symbol $f \in L^{\infty}\left(\mathbb{T}^{n}\right)$. Operating $\mathcal{S}_{w}$ on $\mathcal{S}_{f}$ yields

$$
\mathcal{S}_{w}\left(\mathcal{S}_{f}\right)=\sum_{|\alpha|=0}^{n}(-1)^{|\alpha|} \mathcal{S}_{\varphi_{w}^{\alpha}} \mathcal{S}_{f} \mathcal{S}_{\overline{\varphi_{w}}}{ }^{\alpha}=\sum_{|\alpha|=0}^{n}(-1)^{|\alpha|} \mathcal{S}_{\left|\varphi_{w}^{\alpha}\right|^{2} f}=\mathcal{S}_{\Theta}
$$

with

$$
\Theta=f \sum_{|\alpha|=0}^{n}(-1)^{|\alpha|}\left|\varphi_{w}^{\alpha}\right|^{2}
$$

Replacing both of $z$ and $w$ in Formula $(2.5)$ by $\varphi_{w}(\zeta)$ with $\zeta \in \mathbb{T}^{n}$, we see that

$$
\sum_{|\alpha|=0}^{n}(-1)^{|\alpha|}\left|\varphi_{w}^{\alpha}(z)\right|^{2}=\prod_{j=1}^{n}\left(1-\left|\varphi_{w_{j}}\right|^{2}\right)=0, \text { as each }\left|\varphi_{w_{j}}\right|=1 \text { on the circle } \mathbb{T} \text {. }
$$

Hence, we infer that $\mathcal{S}_{w}\left(\mathcal{S}_{f}\right)=0$ for every dual Toeplitz operator $\mathcal{S}_{f}$.

The following property of the operator $\mathcal{S}_{w}$ will be needed in the sequel:

Theorem 2.1. Let $T$ be a compact operator on $\left(\mathcal{H}^{2}\left(\mathbb{T}^{n}\right)\right)^{\perp}$, then $\left\|\mathcal{S}_{w}(T)\right\| \longrightarrow 0$ as $w \rightarrow \mathbb{T}^{n}$.

Proof. If $\alpha=\left(\alpha_{1}, \alpha_{2}, \ldots, \alpha_{n}\right)$, let us use the notation $\alpha^{\prime}=\left(\alpha_{2}, \ldots, \alpha_{n}\right)$, and observe that:

$$
\begin{aligned}
& \mathcal{S}_{w}(T)=\sum_{|\alpha|=0}^{n}(-1)^{|\alpha|} \mathcal{S}_{\varphi_{w}^{\alpha}} T \mathcal{S}_{\overline{\varphi_{w}}}^{\alpha} \\
& =\sum_{\alpha_{1}, \ldots, \alpha_{n}=0}^{1}(-1)^{|\alpha|} \mathcal{S}_{\varphi_{w_{1}}^{\alpha_{1}}} \cdots \mathcal{S}_{\varphi_{w_{n}}^{\alpha_{n}}} T \mathcal{S}_{\bar{\varphi}_{w_{1}}^{\alpha_{1}}} \cdots \mathcal{S}_{\bar{\varphi}_{w_{n}}^{\alpha_{n}}} \\
& =\sum_{\left|\alpha^{\prime}\right|=0}^{n-1}(-1)^{\left|\alpha^{\prime}\right|} \mathcal{S}_{\varphi_{w_{2}}^{\alpha_{2}}} \cdots \mathcal{S}_{\varphi_{w_{n}}^{\alpha_{n}}}\left(T-\mathcal{S}_{\varphi_{w_{1}}} T \mathcal{S}_{\bar{\varphi}_{w_{1}}}\right) \mathcal{S}_{\bar{\varphi}_{w_{2}}^{\alpha_{2}}} \cdots \mathcal{S}_{\bar{\varphi}_{w_{n}}^{\alpha_{n}}}
\end{aligned}
$$

Hence, we only need to verify that for compact $T$, one has

$$
\left\|T-\mathcal{S}_{\varphi_{w_{1}}} T \mathcal{S}_{\bar{\varphi}_{w_{1}}}\right\| \longrightarrow 0 \text { as } \mathbb{D}^{n} \ni w=\left(w_{1}, \cdots, w_{n}\right) \longrightarrow \zeta=\left(\zeta_{1}, \cdots, \zeta_{n}\right) \in \mathbb{T}^{n} .
$$

Since finite rank operators are dense in the set of compact operators, we only need to verify the latter for rank one operators. For let $f, g \in\left(\mathcal{H}^{2}\left(\mathbb{T}^{n}\right)\right)^{\perp}$; then by (2.6) we get

$$
\begin{aligned}
& \left\|f \otimes g-\mathcal{S}_{\varphi_{w_{1}}}(f \otimes g) \mathcal{S}_{\bar{\varphi}_{w_{1}}}\right\| \\
= & \left\|\left(\zeta_{1} f\right) \otimes\left(\zeta_{1} g\right)-\left(\mathcal{S}_{\varphi_{w_{1}}} f\right) \otimes\left(\mathcal{S}_{\varphi_{w_{1}}} g\right)\right\| \\
\leq & \left\|\left(\zeta_{1} f-\mathcal{S}_{\varphi_{w_{1}}} f\right) \otimes\left(\zeta_{1} g\right)\right\|+\left\|\left(\mathcal{S}_{\varphi_{w_{1}}} f\right) \otimes\left(\zeta_{1} g-\mathcal{S}_{\varphi_{w_{1}}} g\right)\right\| .
\end{aligned}
$$


Now, we know that, for $w_{1} \in \mathbb{D}$ and $\tau \in \mathbb{T}, w_{1}-\varphi_{w_{1}}(\tau) \longrightarrow 0$ a.e. as $\left|w_{1}\right| \rightarrow 1^{-}$. Making appeal to the dominated convergence theorem, we infer that for $f \in\left(\mathcal{H}^{2}\left(\mathbb{T}^{n}\right)\right)^{\perp}$ one has

$$
\left\|w_{1} f-\varphi_{w_{1}} f\right\|_{2}^{2}=\int_{\mathbb{T}^{n}}\left|w_{1} f(\xi)-\varphi_{w_{1}}(\xi) f(\xi)\right|^{2} d \sigma(\xi) \longrightarrow 0 \text { as }\left|w_{1}\right| \longrightarrow 1^{-} .
$$

Hence, we see that $\left\|\zeta_{1} f-\varphi_{w_{1}} f\right\|_{2} \longrightarrow 0$ as $\mathbb{D} \ni w_{1} \longrightarrow \zeta_{1} \in \mathbb{T}$. Because of the identity $(I-\mathcal{P})\left(\zeta_{1} f(\xi)\right)=\zeta_{1} f(\xi)$, we see that

$$
\left\|\zeta_{1} f-\mathcal{S}_{\varphi_{w_{1}}} f\right\|_{2}=\left\|(I-\mathcal{P})\left(\zeta_{1} f-\varphi_{w_{1}} f\right)\right\|_{2} \longrightarrow 0 \text { as } \mathbb{D}^{n} \ni w \longrightarrow \zeta \in \mathbb{T}^{n} .
$$

The latter together with Inequality (2.14) yield:

$$
\left\|f \otimes g-\mathcal{S}_{\varphi_{w_{1}}}(f \otimes g) \mathcal{S}_{\overline{\varphi_{w_{1}}}}\right\| \longrightarrow 0 \text { as } \mathbb{D}^{n} \ni w \longrightarrow \zeta \in \mathbb{T}^{n} .
$$

\section{Commutativity of Dual Toeplitz Operators}

Hermitian dual Toeplitz operators can be characterized quite easily, as the forthcoming lemma shows. However, characterizing normal dual Toeplitz operators is not an immediate task. It is in fact a consequence of our main result in this section as we will see soon.

Lemma 3.1. $\mathcal{S}_{f}$ is self-adjoint if and only if $f$ is real.

Proof. $\mathcal{S}_{f}$ is self-adjoint means that $\mathcal{S}_{f}=\mathcal{S}_{f}^{*}$, which is equivalent to $f=\bar{f}$. Thus, $f$ must be real-valued.

Recall that Lemma 2.1 indicates that $\mathcal{S}_{f}$ and $\mathcal{S}_{g}$ commute if $f$ and $g$ are both analytic or both conjugate analytic. If a non-trivial linear combination of $f$ and $g$ is constant, they also commute. Thus, we are interested to see whether these are the only cases where commutativity takes place. Using similar arguments as in $[14,17]$, we arrive at:

Theorem 3.1. Let $f, g$ be bounded functions on $\mathbb{T}^{n}$. Then, the dual Toeplitz operators $\mathcal{S}_{f}$ and $\mathcal{S}_{g}$ commute on $\left(\mathcal{H}^{2}\left(\mathbb{T}^{n}\right)\right)^{\perp}$, (i.e. $\mathcal{S}_{f} \mathcal{S}_{g}=\mathcal{S}_{g} \mathcal{S}_{f}$ ), if and only if $f$ and $g$ satisfy one of the following conditions:

(1) they are both analytic on $\mathbb{T}^{n}$.

(2) they are both co-analytic on $\mathbb{T}^{n}$.

(3) a non-trivial linear combination of them is constant on $\mathbb{T}^{n}$. 
Proof. The if part is trivial due to Lemma 2.1. With regard to the only if part, observe that by Proposition 2.1 and parts (i) and (ii) of Lemma 2.1 one has

$$
H_{f}\left(k_{w} \otimes k_{w}\right) H_{\bar{g}}^{*}=\sum_{|\alpha|=0}^{n}(-1)^{|\alpha|}\left(\mathcal{S}_{\varphi_{w}^{\alpha}} H_{f}\right)\left(H_{\bar{g}}^{*} \mathcal{S}_{\overline{\varphi_{w}}}{ }^{\alpha}\right)=\mathcal{S}_{w}\left(H_{f} H_{\bar{g}}^{*}\right) .
$$

In a similar wa, we obtain

$$
H_{g}\left(k_{w} \otimes k_{w}\right) H_{f}^{*}=\mathcal{S}_{w}\left(H_{g} H_{\frac{f}{f}}^{*} .\right.
$$

Combining Equations (2.2), (2.6), (3.1) and (3.2), we see that

$$
\left(H_{g} k_{w}\right) \otimes\left(H_{\bar{f}} k_{w}\right)-\left(H_{f} k_{w}\right) \otimes\left(H_{\bar{g}} k_{w}\right)=\mathcal{S}_{w}\left(\left[\mathcal{S}_{f}, \mathcal{S}_{g}\right]\right) .
$$

The assumption reduces the latter to

$$
\left(H_{g} k_{w}\right) \otimes\left(H_{\bar{f}} k_{w}\right)=\left(H_{f} k_{w}\right) \otimes\left(H_{\bar{g}} k_{w}\right), \forall w \in \mathbb{D}^{n} .
$$

In particular, for $w=0$ one has $k_{0}=1$; whence $H_{g} 1 \otimes H_{\bar{f}} 1=H_{f} 1 \otimes H_{\bar{g}} 1$, which can be rewritten as

$$
<h, H_{\bar{f}} 1>H_{g} 1=<h, H_{\bar{g}} 1>H_{f} 1, \forall h \in\left(\mathcal{H}^{2}\left(\mathbb{T}^{n}\right)\right)^{\perp} .
$$

Finally, we distinguish three cases:

(1) If $H_{g} 1 \neq 0$ and $H_{\bar{g}} 1 \neq 0$, then there exists a complex number $\rho \neq 0$ such that $H_{f} 1=\rho H_{g} 1$ and $H_{\bar{f}} 1=\bar{\rho} H_{\bar{g}} 1$. That is to say $\mathcal{Q}(f-\rho g)=\mathcal{Q}(\bar{f}-\overline{\rho g})=0$; whence $f-\rho g$ and $\bar{f}-\overline{\rho g}$ are both analytic. Thus $f-\rho g$ is constant, which corresponds to condition (3).

(2) If $H_{g} 1=0$, then $g$ is analytic. Also we must have either $H_{f} 1=0$ or $H_{\bar{g}} 1=0$, which means that either $f$ is analytic, (which corresponds to condition (1)), or $g$ is co-analytic, (in this case $g$ must be constant, which corresponds to condition (3)).

(3) If $H_{\bar{g}} 1=0$, then $g$ is co-analytic. Also we see that either $H_{g} 1=0$ or $H_{\bar{f}} 1=0$. This means that either $g$ is analytic, (which implies that $g$ is constant and corresponds to condition (3)), or $f$ is co-analytic, (which agrees with condition (2)).

Now, thanks to Theorem 3.1 that normal dual Toeplitz operators can be easily characterized:

Corollary 3.1. Suppose that $f \in L^{\infty}\left(\mathbb{T}^{n}\right)$. Then, the dual Toeplitz operator $\mathcal{S}_{f}$ is normal if and only if the range of its symbol $f$ lies on a line in the complex plane.

Proof. Because $f$ and its conjugate $\bar{f}$ cannot be simultaneously analytic or coanalytic unless $f$ is constant, by Theorem $3.1 \mathcal{S}_{f}$ and $\mathcal{S}_{f}^{*}=\mathcal{S}_{\bar{f}}$ commute if and only if there are constants $\gamma, \beta$ and $\mu$ not all zero such that $\gamma f+\beta \bar{f}=\mu$. Thus, we infer that $\mathcal{S}_{f}$ and $\mathcal{S}_{f}^{*}$ commute if and only if the range of $f$ lies on a line. 


\section{Products of Dual Toeplitz Operators}

For dual Toeplitz operators on Bergman space of the polydisk, a Brown-Halmos theorem has been proved by Y.F. Lu and S.X. Shang in [14]. Our aim in this section is to establish a Brown-Halmos type theorem for our dual Toeplitz operators in an analogous way. Before establishing it, let us first prove the following general form of it. This generalization has been given in a related context first by K. Stroethoff [16] and then also by $\mathrm{C}$. Gu [6] and by Lee [12] as well as by Guediri [7].

Theorem 4.1. Let $f, g, h$ and $k$ be in $L^{\infty}\left(\mathbb{T}^{n}\right)$. Then $S_{f} S_{g}+S_{h} S_{k}$ is a bounded dual Toeplitz operator if and only if one of the following conditions holds

(1) $f$ and $h$ are both analytic.

(2) $g$ and $k$ are both co-analytic.

(3) $f$ is analytic and $k$ is co-analytic.

(4) $h$ is analytic and $g$ is co-analytic.

(5) there is a constant $\gamma \in \mathbb{C} \backslash\{0\}$, such that $h-\gamma f$ is analytic and $g+\gamma k$ is co-analytic.

In all cases $S_{f} S_{g}+S_{h} S_{k}=S_{f g+h k}$.

Proof. The sufficiency of conditions 1, 2, 3 and 4 follows immediately from Lemma 2.1. To prove the sufficiency of condition 5, suppose there exist an analytic function $\varphi$ and a co-analytic one $\psi$ with $h-\gamma f=\phi$, and $g+\gamma k=\psi$. Then, we see that

$$
\begin{aligned}
S_{f} S_{g}+S_{h} S_{k} & =S_{f} S_{(\psi-\gamma k)}+S_{(\varphi+\gamma f)} S_{k} \\
& =S_{f}\left(S_{\psi}-\gamma S_{k}\right)+\left(S_{\varphi}+\gamma S_{f}\right) S_{k} \\
& =S_{f \psi}+S_{k \varphi}=S_{f g+\gamma f k+h k-\gamma f k}=S_{f g+h k}
\end{aligned}
$$

which means that $S_{f} S_{g}+S_{h} S_{k}$ is a dual Toeplitz operator.

To demonstrate the necessity, suppose that $S_{f} S_{g}+S_{h} S_{k}=S_{\vartheta}$, for some $\vartheta \in$ $L^{\infty}\left(\mathbb{T}^{n}\right)$. Using Identities (2.1) we obtain

$$
S_{f g+h k-\vartheta}=H_{f} H_{\bar{g}}^{*}+H_{h} H_{\bar{k}}^{*} .
$$

So, introducing the operator $\mathcal{S}_{w}$, by Proposition 2.1 and parts (i) and (ii) of Lemma 2.1 we observe that

$$
H_{f}\left(k_{w} \otimes k_{w}\right) H_{\bar{g}}^{*}=\sum_{|\alpha|=0}^{n}(-1)^{|\alpha|}\left(\mathcal{S}_{\varphi_{w}^{\alpha}} H_{f}\right)\left(H_{\bar{g}}^{*} \mathcal{S}_{\overline{\varphi_{w}}}^{\alpha}\right)=\mathcal{S}_{w}\left(H_{f} H_{\bar{g}}^{*}\right) .
$$


Similarly, we have

$$
H_{h}\left(k_{w} \otimes k_{w}\right) H_{\bar{k}}^{*}=\mathcal{S}_{w}\left(H_{h} H_{\bar{k}}^{*}\right) .
$$

Combining the three latter identities and owing to Identity (2.6), we see that

$$
\begin{aligned}
\mathcal{S}_{w}\left(S_{f g+h k-\vartheta}\right) & =\mathcal{S}_{w}\left(H_{f} H_{\bar{g}}^{*}\right)+\mathcal{S}_{w}\left(H_{h} H_{\bar{k}}^{*}\right) \\
& =H_{f}\left(k_{w} \otimes k_{w}\right) H_{\bar{g}}^{*}+H_{h}\left(k_{w} \otimes k_{w}\right) H_{\bar{k}}^{*} \\
& =\left(H_{f}\left(k_{w}\right)\right) \otimes\left(H_{\bar{g}}\left(k_{w}\right)\right)+\left(H_{h}\left(k_{w}\right)\right) \otimes\left(H_{\bar{k}}\left(k_{w}\right)\right) .
\end{aligned}
$$

Since $S_{f g+h k-\vartheta}$ is a dual Toeplitz operator, by Proposition 2.2 we infer therefore that:

$$
\left.H_{f}\left(k_{w}\right)\right) \otimes\left(H_{\bar{g}}\left(k_{w}\right)\right)+\left(H_{h}\left(k_{w}\right)\right) \otimes\left(H_{\bar{k}}\left(k_{w}\right)\right)=0 .
$$

In particular, if $w=0 \in \mathbb{T}^{n}$ one gets $k_{0}=1$; so we obtain

$$
H_{f} 1 \otimes H_{\bar{g}} 1=-H_{h} 1 \otimes H_{\bar{k}} 1
$$

Thus, we infer that

$$
\left\langle v, H_{\bar{g}} 1\right\rangle H_{f} 1=-\left\langle v, H_{\bar{k}} 1\right\rangle H_{h} 1, \quad \forall v \in\left(\mathcal{H}^{2}\left(\mathbb{T}^{n}\right)\right)^{\perp} .
$$

Now, we distinguish several cases, (exactly we have $2^{4}=$ sixteen cases):

(1) If $H_{\bar{g}} 1=0$, then one of the following cases must be satisfied:

(a) $H_{f} 1=0, H_{\bar{k}} 1=0$ and $H_{h} 1=0$ (a possible case). This implies that $f$ and $h$ are analytic and $g$ and $k$ are co-analytic. This corresponds to conditions $1,2,3$ and 4 .

(b) $H_{f} 1=0, H_{\bar{k}} 1=0$ and $H_{h} 1 \neq 0$ (a possible case). This implies that $f$ is analytic and $g$ and $k$ are co-analytic; whence conditions 2 and 3 hold.

(c) $H_{f} 1=0, H_{\bar{k}} 1 \neq 0$ and $H_{h} 1=0$ (a possible case). This implies that $f$ and $h$ are analytic and $g$ is co-analytic; whence conditions 1 and 4 hold.

(d) $H_{f} 1=0, H_{\bar{k}} 1 \neq 0$ and $H_{h} 1 \neq 0$ (an impossible case).

(e) $H_{f} 1 \neq 0, H_{\bar{k}} 1 \neq 0$ and $H_{h} 1 \neq 0$ (an impossible case).

(f) $H_{f} 1 \neq 0, H_{\bar{k}^{1}} \neq 0$ and $H_{h} 1=0$ (a possible case). This implies that $h$ is analytic and $g$ is co-analytic; whence condition 4 holds.

(g) $H_{f} 1 \neq 0, H_{\bar{k}} 1=0$ and $H_{h} 1 \neq 0$ (a possible case). This means that $g$ and $k$ are co-analytic; whence condition 2 holds.

(h) $H_{f} 1 \neq 0, H_{\bar{k}} 1=0$ and $H_{h} 1=0$ (a possible case). This means that $h$ is analytic and $g$ and $k$ are co-analytic; whence conditions 2 and 4 hold. 
(2) If else $H_{\bar{g}} 1 \neq 0$, then one of the following cases must be also satisfied:

(a) $H_{f} 1=0, H_{\bar{k}^{1}} 1=0$ and $H_{h} 1=0$ (a possible case). This means that $f$ and $h$ are analytic and $k$ is co-analytic; whence conditions 1 and 3 hold.

(b) $H_{f} 1=0, H_{\bar{k}} 1=0$ and $H_{h} 1 \neq 0$ (a possible case). It implies that $f$ is analytic and $k$ is co-analytic; whence condition 3 holds.

(c) $H_{f} 1=0, H_{\bar{k}} 1 \neq 0$ and $H_{h} 1=0$ (a possible case). This implies that $f$ is analytic and $h$ is analytic; this corresponds to condition 1 .

(d) $H_{f} 1=0, H_{\bar{k}} 1 \neq 0$ and $H_{h} 1 \neq 0$ (an impossible case).

(e) $H_{f} 1 \neq 0, H_{\bar{k}} 1=0$ and $H_{h} 1 \neq 0$ (an impossible case).

(f) $H_{f} 1 \neq 0, H_{\bar{k}} 1=0$ and $H_{h} 1=0$ (an impossible case).

(g) $H_{f} 1 \neq 0, H_{\bar{k}} 1 \neq 0$ and $H_{h} 1=0$ (an impossible case).

(h) $H_{f} 1 \neq 0, H_{\bar{k}} 1 \neq 0$ and $H_{h} 1 \neq 0$ (a possible case). It is in fact the only nontrivial case. We infer that, there is a constant $\rho \in \mathbb{C} \backslash\{0\}$, such that $\rho=-\frac{\left\langle v_{0}, H_{\bar{g}} 1\right\rangle}{\left\langle v_{0}, H_{\bar{k}} 1\right\rangle}$, for some $v_{0} \in\left(\mathcal{H}^{2}\left(\mathbb{T}^{n}\right)\right)^{\perp}$. Thus Equation (4.6) for $v_{0}$ yields $H_{h} 1=\lambda H_{f}$. Substituting the latter in the RHS of Equation (4.6) again, we get $\left\langle v, H_{\bar{g}} 1\right\rangle H_{f} 1=\left\langle v,-\bar{\lambda} H_{\bar{k}} 1\right\rangle H_{f} 1$. Thus, we obtain $H_{\bar{g}} 1=$ $-\bar{\lambda} H_{\bar{k}} 1$; whence, we conclude that $(h-\lambda f) \in\left(\mathcal{H}^{2}\left(\mathbb{T}^{n}\right)\right)^{\perp}$ and $\bar{g}+\overline{\lambda k} \in$ $\left(\mathcal{H}^{2}\left(\mathbb{T}^{n}\right)\right)^{\perp}$. So that, $(h-\lambda f)$ is analytic and $(g+\lambda k)$ is co-analytic; which corresponds to condition 3 .

This discussion completes the proof.

An immediate but interesting corollary about commutators can also be reported, namely

Corollary 4.1. If $f$ and $g$ are in $L^{\infty}\left(\mathbb{T}^{n}\right)$. Then, the commutator $\left[S_{f}, S_{g}\right]$ is a dual Toeplitz operator if and only if $S_{f}$ and $S_{g}$ commute, i.e. $\left[S_{f}, S_{g}\right]=0$.

Proof. Suppose that $S_{f} S_{g}-S_{g} S_{f}$ is a dual Toeplitz operator. Then, from Theorem 4.1 we see that one of the following conditions holds

(1) $f$ and $g$ are analytic.

(2) $f$ and $g$ are co-analytic.

(3) $f$ is constant.

(4) $g$ is constant.

(5) there is a constant $\gamma \in \mathbb{C} \backslash\{0\}$, such that $g+\gamma f$ is constant. 
Hence by Theorem 3.1 we see that $S_{f}$ and $S_{g}$ commute.

Conversely, if $S_{f}$ and $S_{g}$ commute, then $S_{f} S_{g}-S_{g} S_{f}=0=S_{0}$; which is the trivial dual Toeplitz operator.

Now, we are in the position to state our main result in this section, namely the Brown-Halmos theorem; which can now be obtained as a corollary from Theorem 4.1 by taking $h \equiv k \equiv 0$ :

Theorem 4.2. Let $f$ and $g$ be in $L^{\infty}\left(\mathbb{T}^{n}\right)$. Then, the dual Toeplitz product $\mathcal{S}_{f} \mathcal{S}_{g}$ is again a dual Toeplitz operator if and only if one of the following conditions holds:

(1) $f$ is analytic.

(2) $g$ is co-analytic.

In either cases $\mathcal{S}_{f} \mathcal{S}_{g}=\mathcal{S}_{f g}$.

A first corollary is about the so-called zero product problem. It tells us that among the class of dual Toeplitz operators on the orthogonal complement of the Hardy space of the polydisk there are no zero divisors.

Corollary 4.2. The product $\mathcal{S}_{f} \mathcal{S}_{g}$ of two dual Toeplitz operators on $\left(\mathcal{H}^{2}\left(\mathbb{T}^{n}\right)\right)^{\perp}$ is zero if and only if one of the symbols $f$ or $g$ is zero.

Proof. If $\mathcal{S}_{f}=0$ or $\mathcal{S}_{g}=0$, then immediately $\mathcal{S}_{f} \mathcal{S}_{g}=0$. Conversely, assume that $\mathcal{S}_{f} \mathcal{S}_{g}=0$. Then $\mathcal{S}_{f} \mathcal{S}_{g}$ is a dual Toeplitz operator with symbol zero. Theorem 4.2 implies that either $f$ is analytic or $g$ is co-analytic and moreover $\mathcal{S}_{f} \mathcal{S}_{g}=\mathcal{S}_{f g}=0$. Thus $f g=0$ a.e. on $\mathbb{T}^{n}$. We then have two cases:

(1) If $f$ is analytic. Then, in case $g=0$ a.e., the result follows. But if $g \neq 0$, then $f$ must vanish on a subset of positive measure; whence $f \equiv 0$ on $\mathbb{T}^{n}$.

(2) If $g$ is co-analytic. Then, in case $f=0$ a.e., the result follows. But if $f \not \equiv 0$, then the analytic function $\bar{g}$ must vanish on a subset of positive measure; whence $\bar{g} \equiv 0$, and thus $g$ vanishes on $\mathbb{T}^{n}$.

We conclude that either $\mathcal{S}_{f}=0$ or $\mathcal{S}_{g}=0$.

Corollary 4.3. Let $f, g$ and $h$ be in $L^{\infty}\left(\mathbb{T}^{n}\right)$ with $f \not \equiv 0$, such that $\mathcal{S}_{f} \mathcal{S}_{g}=\mathcal{S}_{f} \mathcal{S}_{h}$. Then, we must have $g=h$.

Proof. Because $\mathcal{S}_{f} \mathcal{S}_{g}=\mathcal{S}_{f} \mathcal{S}_{h}$, we get $\mathcal{S}_{f}\left(\mathcal{S}_{g-h}\right)=0$. Using Corollary 4.2, we infer that $f(g-h)=0$; whence $g=h$.

Corollary 4.4. The only idempotent dual Toeplitz operators are the trivial ones, (0 or $I)$. 
Proof. $\quad$ If $\mathcal{S}_{f}^{2}=\mathcal{S}_{f}$, then $\mathcal{S}_{f}^{2}-\mathcal{S}_{f}=\mathcal{S}_{f}\left(\mathcal{S}_{f}-I\right)=\mathcal{S}_{f}\left(\mathcal{S}_{f}-\mathcal{S}_{1}\right)=\mathcal{S}_{f} \mathcal{S}_{f-1}=0$. By Corollary 4.2, we get $\mathcal{S}_{f}=0$ or $\mathcal{S}_{f-1}=0$. Therefore $\mathcal{S}_{f}=0$ or $\mathcal{S}_{f}=\mathcal{S}_{1}=I$.

Corollary 4.5. A dual Toeplitz operator $\mathcal{S}_{f}$ is an isometry if and only if $f$ is co-analytic in $\mathbb{D}^{n}$ and unimodular on $\mathbb{T}^{n}$.

Proof. If $\mathcal{S}_{f}$ is an isometry, then $\mathcal{S}_{\bar{f}} \mathcal{S}_{f}=\mathcal{S}_{1}$. Thus, Theorem 4.2 implies that $f$ must be co-analytic. Moreover, we should have $\bar{f} f=|f|^{2}=1$ on $\mathbb{T}^{n}$.

Conversely if $f$ is a co-analytic function of boundary modulus 1 then it is clear that $\mathcal{S}_{f}^{*} \mathcal{S}_{f}=\mathcal{S}_{\bar{f}} \mathcal{S}_{f}=\mathcal{S}_{|f|^{2}}=I$. Therefore, $\mathcal{S}_{f}$ is an isometry; and the proof is complete.

Corollary 4.6. A dual Toeplitz operator $\mathcal{S}_{f}$ is unitary if and only if $f$ is a unimodular constant function.

Proof. If $\mathcal{S}_{f}$ is unitary, then $\mathcal{S}_{f}^{*} \mathcal{S}_{f}=\mathcal{S}_{f} \mathcal{S}_{f}^{*}=I$, i.e. $\mathcal{S}_{\bar{f}} \mathcal{S}_{f}=\mathcal{S}_{f} \mathcal{S}_{\bar{f}}=\mathcal{S}_{1}$. Thus, Theorem 4.2 implies that $f$ must be simultaneously analytic and co-analytic; whence constant in $\mathbb{D}^{n}$. Besides, we should have $\bar{f} f=|f|^{2}=1$.

Conversely, if $f$ is a constant function of modulus 1 , then $\mathcal{S}_{f}=\lambda I$ for some unimodular complex constant $\lambda$; whence $\mathcal{S}_{f}^{*} \mathcal{S}_{f}=\mathcal{S}_{f} \mathcal{S}_{f}^{*}=I$. Therefore, $\mathcal{S}_{f}$ is unitary; which completes the proof.

Corollary 4.7. Suppose that the dual Toeplitz operator $\mathcal{S}_{f}$ is invertible, and that its inverse $\mathcal{S}_{f}^{-1}$ is again a dual Toeplitz operator. Then, $f$ must be either analytic or co-analytic.

Proof. Suppose that $\mathcal{S}_{f}^{-1}$ is a dual Toeplitz operator $\mathcal{S}_{g}$ for some bounded symbol $g$ say. Since $\mathcal{S}_{f}^{-1} \mathcal{S}_{f}=\mathcal{S}_{g} \mathcal{S}_{f}=I=\mathcal{S}_{1}$, which is a dual Toeplitz operator, Theorem 4.2 implies, on the one hand, that either $f$ is co-analytic or $g$ is analytic. On the other hand since we have $\mathcal{S}_{f} \mathcal{S}_{f}^{-1}=\mathcal{S}_{f} \mathcal{S}_{g}=I=\mathcal{S}_{1}$, so again by Theorem 4.2, we see that either $g$ is co-analytic or $f$ is analytic. Now, if $f$ is analytic then we are done. But if $f$ is not analytic, then $g$ must be co-analytic and non-constant (because if $g$ is constant then $\mathcal{S}_{g}=\mathcal{S}_{f}^{-1}=\lambda I$ which means that $\mathcal{S}_{f}=\frac{1}{\lambda} I$, i.e. $f=\frac{1}{\lambda}$ which is analytic). Thus $g$ is not analytic and hence $f$ must be co-analytic (by the first case), which completes the proof.

\section{Hankel Products and Mixed Toeplitz-hankel Products}

In this section, we make use of the above pioneering operator $\mathcal{S}_{w}$ in order to establish necessary conditions for boundedness and compactness of Hankel products and mixed Toeplitz-Hankel products on the Hardy space of the polydisk. This problem in the framework of the Bergman space has been studied by Y.F. Lu and S.X. Shang in [13], which is in fact our main reference in this section; for the same problem in related contexts we refer to $[8,11]$.

The following assertion provides a necessary condition for the boundedness of a Hankel product $H_{f} H_{g}^{*}$ : 
Theorem 5.1. Suppose that $f, g \in L^{2}\left(\mathbb{T}^{n}\right)$. If the Hankel product $H_{f} H_{g}^{*}$ is bounded on $\left(\mathcal{H}^{2}\left(\mathbb{T}^{n}\right)\right)^{\perp}$, then

$$
\sup _{w \in \mathbb{D}^{n}}\left\|f \circ \varphi_{w}-\mathcal{P}\left(f \circ \varphi_{w}\right)\right\|_{2}\left\|g \circ \varphi_{w}-\mathcal{P}\left(g \circ \varphi_{w}\right)\right\|_{2}<\infty .
$$

Proof. Combining Proposition 1 of Stroethoff \& Zheng [18] and the variable change formula (Corollary 1.2) of Ding [5], we obtain

$$
\left\|H_{f} k_{w}\right\|_{2}\left\|H_{g} k_{w}\right\|_{2}=\left\|f \circ \varphi_{w}-\mathcal{P}\left(f \circ \varphi_{w}\right)\right\|_{2}\left\|g \circ \varphi_{w}-\mathcal{P}\left(g \circ \varphi_{w}\right)\right\|_{2} .
$$

By the above norm formula of rank one operators and Equation (2.6), we have

$$
\left\|H_{f} k_{w}\right\|_{2}\left\|H_{g} k_{w}\right\|_{2}=\left\|\left(H_{f} k_{w}\right) \otimes\left(H_{g} k_{w}\right)\right\|=\left\|H_{f}\left(k_{w} \otimes k_{w}\right) H_{g}^{*}\right\| .
$$

Thus, it suffices to verify that the R.H.S. of the latter is bounded. Since $\varphi_{w} \in \mathcal{H}^{\infty}\left(\mathbb{T}^{n}\right)$, we see by Lemma 2.1 that $H_{f} T_{\varphi_{w}}=\mathcal{S}_{\varphi_{w}} H_{f}$ and $T_{\overline{\varphi_{w}}} H_{g}^{*}=H_{g}^{*} \mathcal{S}_{\overline{\varphi_{w}}}$. Thus in a similar way to Identity (3.1), inserting $H_{f}$ and $H_{g}^{*}$ into Formula (2.7), we obtain the following formula

$$
H_{f}\left(k_{w} \otimes k_{w}\right) H_{g}^{*}=\sum_{|\alpha|=0}^{n}(-1)^{|\alpha|} \mathcal{S}_{\varphi_{w}^{\alpha}}\left(H_{f} H_{g}^{*}\right) \mathcal{S}_{\overline{\varphi_{w}}}{ }^{\alpha} .
$$

On the other hand, we have $\left\|\mathcal{S}_{\bar{\varphi}_{w}} \alpha=\right\| \mathcal{S}_{\varphi_{w}^{\alpha}}\|\leq\| \varphi_{w}^{\alpha} \|_{\infty} \leq 1$. Thus, we infer that

$$
\left\|H_{f}\left(k_{w} \otimes k_{w}\right) H_{g}^{*}\right\| \leq \sum_{|\alpha|=0}^{n}\left\|\mathcal{S}_{\varphi_{w}^{\alpha}}\right\|\left\|H_{f} H_{g}^{*}\right\|\left\|\mathcal{S}_{\overline{\varphi_{w}}} \alpha\right\| \leq \sum_{|\alpha|=0}^{n}\left\|H_{f} H_{g}^{*}\right\|<\infty ;
$$

whence, the theorem is proved.

The following result gives a necessary condition for the compactness of a Hankel product $H_{f} H_{g}^{*}$.

Theorem 5.2. Let $f$ and $g$ be in $L^{2}\left(\mathbb{T}^{n}\right)$. If the Hankel product $H_{f} H_{g}^{*}$ is compact, then

$$
\lim _{w \rightarrow \mathbb{T}^{n}}\left\|f \circ \varphi_{w}-\mathcal{P}\left(f \circ \varphi_{w}\right)\right\|_{2}\left\|g \circ \varphi_{w}-\mathcal{P}\left(g \circ \varphi_{w}\right)\right\|_{2}=0 .
$$

Proof. By Equations (5.2), (5.3) and (5.4), we see that

$$
\left\|f \circ \varphi_{w}-\mathcal{P}\left(f \circ \varphi_{w}\right)\right\|_{2}\left\|g \circ \varphi_{w}-\mathcal{P}\left(g \circ \varphi_{w}\right)\right\|_{2}=\left\|\mathcal{S}_{w}\left(H_{f} H_{g}^{*}\right)\right\| .
$$

Hence, if $H_{f} H_{g}^{*}$ is compact, by Theorem 2.1 we infer that

$$
\lim _{w \rightarrow \mathbb{T}^{n}}\left\|\mathcal{S}_{w}\left(H_{f} H_{g}^{*}\right)\right\|=0
$$

which completes the proof of the assertion.

Owing to the alternative representation (2.2) of the commutator of two dual Toeplitz operators, we can characterize its compactness: 
Theorem 5.3. Let $f$ and $g$ be bounded measurable on $\mathbb{T}^{n}$. If the commutator $\left[\mathcal{S}_{f}, \mathcal{S}_{g}\right]$ is compact, then

$$
\left\|\left(H_{g} k_{w}\right) \otimes\left(H_{\bar{f}} k_{w}\right)-\left(H_{f} k_{w}\right) \otimes\left(H_{\bar{g}} k_{w}\right)\right\| \longrightarrow 0 \text { as }|w| \rightarrow 1^{-} .
$$

Proof. Making use of Formulas (2.2) and (5.4), we obtain:

$$
\mathcal{S}_{w}\left(\left[\mathcal{S}_{f}, \mathcal{S}_{g}\right]\right)=\left(H_{g} k_{w}\right) \otimes\left(H_{\bar{f}} k_{w}\right)-\left(H_{f} k_{w}\right) \otimes\left(H_{\bar{g}} k_{w}\right) .
$$

So, if the commutator is compact, then the result follows from Theorem 2.1.

Analog characterizations of bounded and compact mixed Toeplitz-Hankel products $T_{f} H_{g}^{*}$ and $H_{g} T_{f}$ can also be obtained:

Theorem 5.4. Let $f$ be in $\mathcal{H}^{2}\left(\mathbb{T}^{n}\right)$ and $g$ be in $L^{2}\left(\mathbb{T}^{n}\right)$. If one of the mixed Haplitz products $T_{f} H_{g}^{*}$ or $H_{g} T_{\bar{f}}$ is bounded, then

$$
\sup _{w \in \mathbb{D}^{n}}\left\|f \circ \varphi_{w}\right\|_{2}\left\|g \circ \varphi_{w}-\mathcal{P}\left(g \circ \varphi_{w}\right)\right\|_{2}<\infty .
$$

Proof. Relying on the fact that $\varphi_{w} \in \mathcal{H}^{\infty}\left(\mathbb{T}^{n}\right)$ and owing to the analyticity of $f$, we see by Lemma 2.1 that $T_{f} T_{\varphi_{w}}=T_{\varphi_{w}} T_{f}$ and $T_{\overline{\varphi_{w}}} H_{g}^{*}=H_{g}^{*} \mathcal{S}_{\overline{\varphi_{w}}}$. Thus, as in the proof of Theorem 5.1, inserting $T_{f}$ and $H_{g}^{*}$ into Formula (2.7), we see that

$$
T_{f}\left(k_{w} \otimes k_{w}\right) H_{g}^{*}=\sum_{|\alpha|=0}^{n}(-1)^{|\alpha|} T_{\varphi_{w}^{\alpha}}\left(T_{f} H_{g}^{*}\right) \mathcal{S}_{\bar{\varphi}_{w}}^{\alpha} .
$$

Estimating the norms of Toeplitz and dual Toeplitz operators with automorphic symbols, we get $\left\|T_{\varphi_{w}^{m}}\right\| \leq 1$ and $\left\|\mathcal{S}_{\overline{\varphi_{w}}}^{m}\right\| \leq 1$. Thus, if $T_{f} H_{g}^{*}$ is bounded, we infer that

$$
\left\|T_{f}\left(k_{w} \otimes k_{w}\right) H_{g}^{*}\right\| \leq \sum_{|\alpha|=0}^{n}\left\|T_{f} H_{g}^{*}\right\|<\infty .
$$

Hence, as in Equations (5.2) and (5.3), we obtain the claimed estimate. Similar argument can be used to handel the second case.

Compact mixed Haplitz products can also be characterized similarly:

Theorem 5.5. Let $f \in \mathcal{H}^{\infty}\left(\mathbb{T}^{n}\right)$ and $g \in L^{2}\left(\mathbb{T}^{n}\right)$. If one of the mixed Haplitz products $T_{f} H_{g}^{*}$ or $H_{g} T_{\bar{f}}$ is compact, then

$$
\lim _{w \rightarrow \mathbb{T}^{n}}\left\|f \circ \varphi_{w}\right\|_{2}\left\|g \circ \varphi_{w}-\mathcal{P}\left(g \circ \varphi_{w}\right)\right\|_{2}=0 .
$$


Proof. $\quad$ As in the proof of Theorem 2.1, for any operator $A:\left(\mathcal{H}^{2}\left(\mathbb{T}^{n}\right)\right)^{\perp} \longrightarrow$ $\mathcal{H}^{2}\left(\mathbb{T}^{n}\right)$, we have

$$
\begin{aligned}
& \sum_{|\alpha|=0}^{n}(-1)^{|\alpha|} T_{\varphi_{w}^{\alpha}} A \mathcal{S}_{\bar{\varphi}_{w}}^{\alpha} \\
= & \sum_{\left|\alpha^{\prime}\right|=0}^{n-1}(-1)^{\left|\alpha^{\prime}\right|} T_{\varphi_{w_{2}}^{\alpha_{2}}} \cdots T_{\varphi_{w_{n}}^{\alpha_{n}}}\left(A-T_{\varphi_{w_{1}}} A \mathcal{S}_{\bar{\varphi}_{w_{1}}}\right) \mathcal{S}_{\bar{\varphi}_{w_{2}}^{\alpha_{2}}} \cdots \mathcal{S}_{\bar{\varphi}_{w_{n}}^{\alpha_{n}}} .
\end{aligned}
$$

We claim that if such a $A$ is compact, then

$$
\lim _{w \rightarrow \mathbb{T}^{n}} \sum_{|\alpha|=0}^{n}(-1)^{|\alpha|} T_{\varphi_{w}^{\alpha}} A \mathcal{S}_{\overline{\varphi_{w}}}^{\alpha}=0
$$

By Identity (5.10), we only need to verify that

$$
\| A-T_{\varphi_{w_{1}}} A \mathcal{S}_{\overline{\varphi_{w_{1}}}} \mid \longrightarrow 0 \text { as }\left|w_{1}\right| \longrightarrow 1^{-} .
$$

Using the density of finite rank operators in the set of compact operators, we only need to verify the latter for rank one operators acting from $\left(\mathcal{H}^{2}\left(\mathbb{T}^{n}\right)\right)^{\perp}$ into $\mathcal{H}^{2}\left(\mathbb{T}^{n}\right)$. For let $f \in \mathcal{H}^{2}\left(\mathbb{T}^{n}\right)$ and $g \in\left(\mathcal{H}^{2}\left(\mathbb{T}^{n}\right)\right)^{\perp}$. Then, one has

$$
\begin{aligned}
& \left\|f \otimes g-T_{\varphi_{w_{1}}}(f \otimes g) \mathcal{S}_{\overline{\varphi_{w_{1}}}}\right\| \\
\leq & \left\|\left(\zeta_{1} f-T_{\varphi_{w_{1}}} f\right) \otimes\left(\zeta_{1} g\right)\right\|+\left\|\left(T_{\varphi_{w_{1}}} f\right) \otimes\left(\zeta_{1} g-\mathcal{S}_{\varphi_{w_{1}}} g\right)\right\| . \\
= & \left\|\zeta_{1} f-T_{\varphi_{w_{1}}} f\right\|_{2}\left\|\zeta_{1} g\right\|_{2}+\left\|T_{\varphi_{w_{1}}} f\right\|_{2}\left\|\zeta_{1} g-\mathcal{S}_{\varphi_{w_{1}}} g\right\|_{2} .
\end{aligned}
$$

Now, for $\tau \in \mathbb{T}$ and $w_{1} \in \mathbb{D}$, observe that $w_{1}-\varphi_{w_{1}}(\tau) \longrightarrow 0$ a.e. as $\left|w_{1}\right| \rightarrow 1^{-}$. Making use of the dominated convergence theorem, we infer that for $f \in \mathcal{H}^{2}\left(\mathbb{T}^{n}\right)$ and $g \in\left(\mathcal{H}^{2}\left(\mathbb{T}^{n}\right)\right)^{\perp}$ one has

$$
\left\|w_{1} f-\varphi_{w_{1}} f\right\|_{2}^{2}=\int_{\mathbb{T}^{n}}\left|w_{1} f(\xi)-\varphi_{w_{1}}(\xi) f(\xi)\right|^{2} d \sigma(\xi) \longrightarrow 0 \text { as }\left|w_{1}\right| \longrightarrow 1^{-},
$$

and

$$
\left\|w_{1} g-\varphi_{w_{1}} g\right\|_{2}^{2}=\int_{\mathbb{T}^{n}}\left|w_{1} g(\xi)-\varphi_{w_{1}}(\xi) g(\xi)\right|^{2} d \sigma(\xi) \longrightarrow 0 \text { as }\left|w_{1}\right| \longrightarrow 1^{-} .
$$

Hence, we see that $\left\|\zeta_{1} f-\varphi_{w_{1}} f\right\|_{2} \longrightarrow 0$ and $\left\|\zeta_{1} g-\varphi_{w_{1}} g\right\|_{2} \longrightarrow 0$ as $\mathbb{D} \ni w_{1} \longrightarrow$ $\zeta_{1} \in \mathbb{T}$. Because of the identities $\mathcal{P}\left(\zeta_{1} f(\xi)\right)=\zeta_{1} f(\xi)$ and $(I-\mathcal{P})\left(\zeta_{1} g(\xi)\right)=\zeta_{1} g(\xi)$, we see that

$$
\left\|\zeta_{1} f-T_{\varphi_{w_{1}}} f\right\|_{2}=\left\|\mathcal{P}\left(\zeta_{1} f-\varphi_{w_{1}} f\right)\right\|_{2} \longrightarrow 0 \text { as } \mathbb{D}^{n} \ni w \longrightarrow \zeta \in \mathbb{T}^{n},
$$


and

$$
\left\|\zeta_{1} g-\mathcal{S}_{\varphi_{w_{1}}} g\right\|_{2}=\left\|(I-\mathcal{P})\left(\zeta_{1} g-\varphi_{w_{1}} g\right)\right\|_{2} \longrightarrow 0 \text { as } \mathbb{D}^{n} \ni w \longrightarrow \zeta \in \mathbb{T}^{n} .
$$

Combining the latter two limits together with Inequality (5.13), we infer that

$$
\left\|f \otimes g-T_{\varphi_{w_{1}}}(f \otimes g) \mathcal{S}_{\overline{\varphi_{w_{1}}}}\right\| \longrightarrow 0 \text { as } \mathbb{D}^{n} \ni w \longrightarrow \zeta \in \mathbb{T}^{n} ;
$$

which proves (5.11).

Next, suppose for instance that $T_{f} H_{g}^{*}$ is compact, (the other case related to $H_{g} T_{\bar{f}}$, can be handled similarly), then by (5.8) and (5.11), we see that

$$
\left\|T_{f}\left(k_{w} \otimes k_{w}\right) H_{g}^{*}\right\| \longrightarrow 0 \text { as } \mathbb{D}^{n} \ni w \longrightarrow \zeta \in \mathbb{T}^{n} .
$$

Thus, as in Equations (5.2) and (5.3), we obtain the claimed condition.

\section{ACKNOWLEDGMENT}

The authors would like to thank the referee for the useful comments. The second author would like to thank both of King Saud University, Riyadh, Saudi Arabia and the International Center For Theoretical Physics (ICTP, Trieste, Italy) for their invaluable support.

\section{REFERENCES}

1. P. Ahern and Ž. Čučkovié, A theorem of Brown-Halmos type for Bergman space Toeplitz operators, J. Funct. Anal., 187 (2001), 200-210.

2. S. Axler and Ž. Čučković, Commuting Toeplitz operators with harmonic symbols, Integr. Equat. Oper. Th., 14 (1991), 1-12.

3. A. Brown and P. R. Halmos, Algebraic properties of Toeplitz operators, J. Reine Angew. Math., 213 (1963/1964), 89-102.

4. Y. Chen and T. Yu, Essentially commuting dual Toeplitz operators on the unit ball, $A d v$. Math. (China), 38(4) (2009), 453-464.

5. X. H. Ding, Products of Toeplitz operators on the polydisk, Integr. Equ. Oper. Theory, 45 (2003), 389-403.

6. C. Gu, Some algebraic properties of Toeplitz and Hankel operators on polydisk, Arch. Math., 80 (2003), 393-405.

7. H. Guediri, Dual Toeplitz operators on the sphere, Acta Mathematica Sinica, English Series, 29(9) (2013), 1791-1808.

8. H. Guediri, Products of Toeplitz and Hankel operators on the Hardy space of the unit sphere, Operator Theory: Advances and Applications, 236 (2014), 243-256. 
9. H. Guediri, Quasinormality and numerical ranges of certain classes of dual Toeplitz operators, Abstract and Applied Analysis, 2010 (2010), Article ID 426319, doi:10.1155/2010/ 426319, 14 pages.

10. H. Guediri, New function theoretic proofs of Brown-Halmos theorems, Arab J. Math. Sc., 13 (2007), 15-26.

11. M. Hamada, Remark on application of distribution function inequality for Toeplitz and Hankel operators, Hokkaido Math. J., 32 (2003), 193-208.

12. Y. J. Lee, Commuting Toeplitz operators on the Hardy space of the polydisk, Proc. Amer. Math. Soc., 138(1) (2010), 189-197.

13. Y. F. Lu and S. X. Shang, Bounded Hankel products on the Bergman space of the polydisk, Cand. J. Math., 61(1) (2009), 190-204.

14. Y. F. Lu and S. X. Shang, Commuting dual Toeplitz operators on the polydisk, Acta Math. Sinica (Eng. Ser.), 23(5) (2006), 857-868.

15. W. Rudin, Function Theory in the Polydiscs, W. A. Benjamin, New York, 1969.

16. K. Stroethoff, Algebraic properties of Toeplitz operators on the Hardy space via the Berezin transform, Contemporary Math., 232 (1999), 313-319.

17. K. Stroethoff and D. Zheng, Algebraic and spectral properties of dual Toeplitz operators, Trans. Amer. Math. Soc., 354(6) (2002), 2495-2520.

18. K. Stroethoff and D. Zheng, Toeplitz and Hankel operators on Bergman spaces, Trans. Amer. Math. Soc., 329(2) (1992), 773-794.

Lakhdar Benaissa

Faculty of Science

Department of Mathematics

Ferhat Abbas University-Setif 1

Setif 19000

Algeria

and

ESC, Ecole supérieure de Commerce

Algiers 16000

Algeria

E-mail: lakhdar.benaissa@gmail.com

Hocine Guediri

Department of Mathematics

College of Science

King Saud University

P. O. Box 2455

Riyadh 11451

Saudi Arabia

E-mail: hguediri@ksu.edu.sa 